\title{
An Overview of the Application of Deep Learning in Short-Read Sequence Classification
}

\author{
Kristaps Bebris ${ }^{1}$, Inese Polaka ${ }^{2}$ \\ ${ }^{1}$ University of Latvia, Riga, Latvia \\ ${ }_{1,2}^{2}$ Riga Technical University, Riga, Latvia
}

\begin{abstract}
Advances in sequencing technology have led to an ever increasing amount of available short-read sequencing data. This has, consequently, exacerbated the need for efficient and precise classification tools that can be used in the analysis of these data. As it stands, recent years have shown that massive leaps in performance can be achieved when it comes to approaches that are based on heuristics, and apart from these improvements there has been an ever increasing interest in applying deep learning techniques to revolutionize this classification task. We attempt to study these approaches and to evaluate their performance in a reproducible fashion to get a better perspective on the current state of deep learning based methods when it comes to the classification of short-read sequencing data.
\end{abstract}

Keywords - Bioinformatics, computational biology, machine learning.

\section{INTRODUCTION}

Individual genome and metagenome sequencing has become increasingly more affordable over the past years [1], [2], which has led to an explosion in the amount of data available for analysis. This has, in turn, spawned a need for more affordable tools to analyse these data. In the present study, we will consider the recent taxonomic classification tools and evaluate their performance when working with metagenome sequencing data. Kraken2, a state-of-the-art tool widely used for such tasks, has shown that there is still a lot of room for improvement in currently used methods. Kraken 2 allows reducing its memory footprint to just $15 \%$ and its runtime to just $20 \%$ compared to its predecessor Kraken [3].

A number of deep learning and machine learning based tools have been developed in recent years with a goal of alleviating different and resource intensive aspects of current methods memory requirements, classification time, classification precision or disk space requirements [4]-[7]. A major issue of evaluating the current state of these tools lies in the fact that there are no published results showing their relative performance when the same reference data are used and when they are applied to non-synthetic samples. This is an issue that is characteristic of many bioinformatics tools [8]. There is also a known issue with self-reported performance metrics, which are the only results available for a lot of tools - they tend to have bias problems [9].

There have been some attempts to standardise the way tools are benchmarked [8], but so far no standard protocols nor benchmark data sets have been adopted and most tools still report their results using bespoke approaches. This makes gauging the performance of these tools in real world scenarios quite tricky as generated samples can be less complex than real ones [16].

Our goal is to evaluate the performance of a set of freely available published deep learning based tools by using the exact same reference data: metagenome data from a set of samples that have been sequenced by MGI DNBSEQ-T7 sequencers [10], which include two reference samples with a predefined composition (Zymo samples [11] - referred to as C1 and C2) and three human gut (fecal) metagenome samples that were produced within the ERDF project "Optimisation of H.pylori Eradication Therapy for Population-Based Gastric Cancer Prevention" (referred to as S1, S97 and S104). The non-control samples were added to the experiment to assess how the tools would perform if the reference database did not contain all of the organisms present in the sample and therefore there were no exact matches to the sequences in the sample.

\section{METHODS}

We selected suitable deep learning based tools for genomic classification by searching the SCOPUS and Arxiv databases, excluding the tools that were designed for tasks other than shotgun sequence classification. This way we chose four tools:

- MetaVW - embedding based tool that leverages Vowpal Wabbit [12] as its backbone [4];

- fastDNA - fastText [18] based embedding tool [5];

- GeNet - a classification tool that uses a convolutional model [6];

- DeepMicrobes - a classification tool that leverages an attention model [7].

In addition, we decided to use the Kraken2 results as our 'ground truth' for samples that we did not have a theoretical taxonomic distribution because it was a widely recognised and commonly used tool [3]. We found this necessesary due to the way the experiment was set up: we included real-life samples with an unknown composition, so an estimate of their composition was established using Kraken2 as a reliable stateof-the-art tool.

The expirements were performed on a workstation running a 12-core procesor (Intel i7-8700k), 64 GB of RAM, Nvidia GTX 1080 Ti graphics card (GPU) and 500 GB of PCIe storage. We 
make a point of noting down the storage solution due to Vowpal Wabbit being heavily bound by storage speed [12] that will impact the performance of MetaVW [4].
Reference databases were created and models were trained using the default setting wherever possible. The only deviation from this being DeepMicrobes where we used 11-mers instead

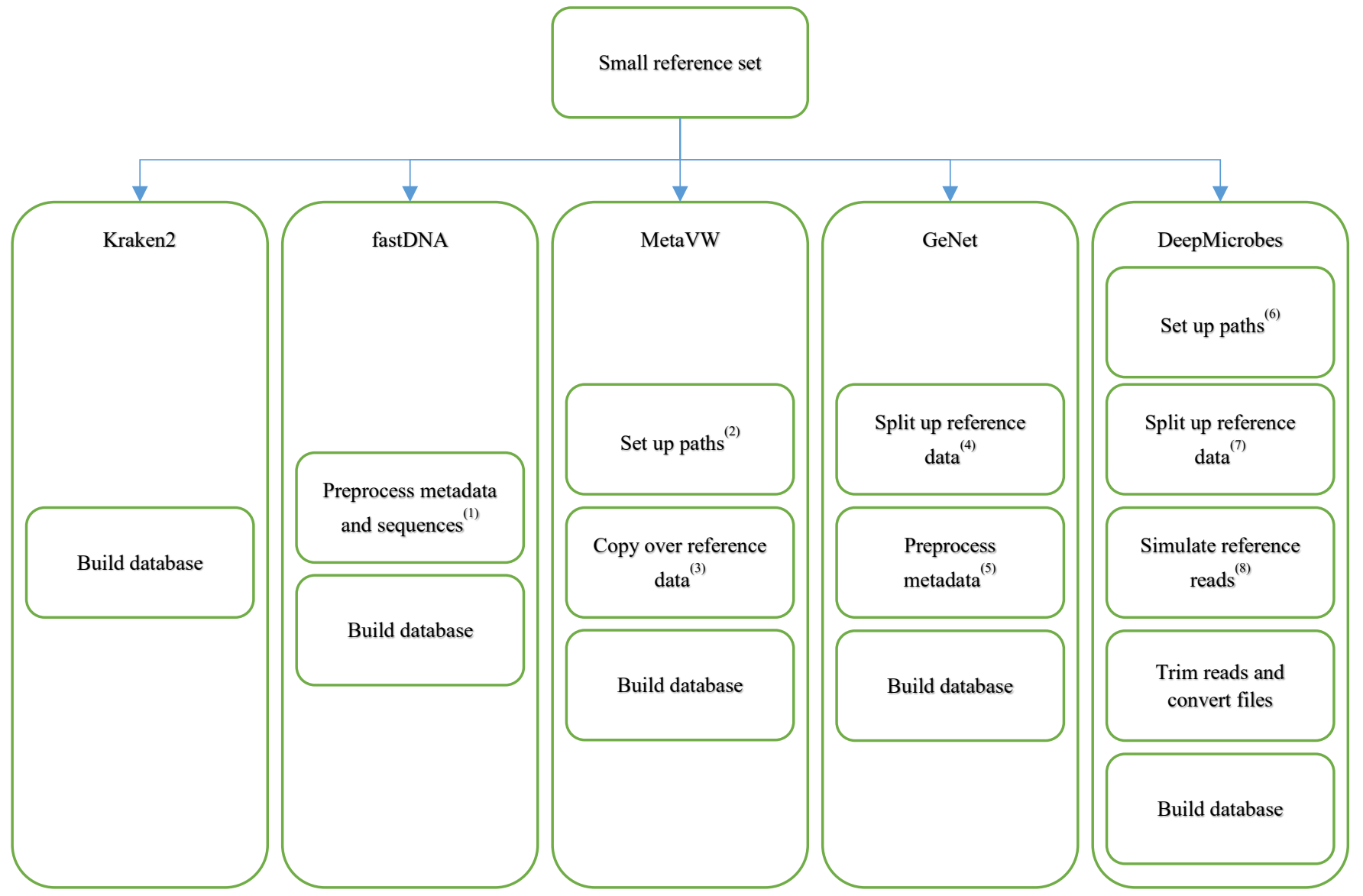

Fig. 1. An overall outline of the database creation process. Some practical items that we would like to draw additional attention to include: fastDNA (1) requires all sequences to be uppercase and needs custom meta tags for the sequences, MetaVW (2) requires some external libraries that the tool does not ship with, GeNet (3) needs the reference data to be split up on a taxon-by-taxon basis and (4) requires custom meta tags for the sequences, DeepMicrobes (5) ships with all of the required tools, but they are split up between a multitude of subdirectories, (6) it also requires the reference data to be split up into separate files similarily to GeNet and (7) the training set has to be processed with a read simulator [18]. The scripts that this figure represents are available on GitHub [14].

We evaluated all tools using the same five samples (read counts and average read length, which provide insight into data complexity, are given in Table I) and used the small database from MetaVW [13] (mostly due to practical concerns - it contained all of the information needed for all of the tools to train) to create the reference databases for all of the tools (containing 1565 sequences related to 193 species) [4].

The two control samples are ZymoBIOMICSTM Microbial Community Standard samples that contain data from ten different species [11].

TABLE I

SAMPLE CHARACTERISTICS

\begin{tabular}{|l|c|c|c|c|}
\hline Sample & $\begin{array}{c}\text { Forward read } \\
\text { count }\end{array}$ & $\begin{array}{c}\text { Forward } \\
\text { average read } \\
\text { length }\end{array}$ & $\begin{array}{c}\text { Reverse read } \\
\text { count }\end{array}$ & $\begin{array}{c}\text { Reverse } \\
\text { average read } \\
\text { length }\end{array}$ \\
\hline C1 & 38581675 & 150 & 38581675 & 150 \\
\hline C2 & 52537916 & 150 & 52537916 & 150 \\
\hline S1 & 28990760 & 150 & 28990760 & 150 \\
\hline S97 & 28987851 & 150 & 28987851 & 150 \\
\hline S104 & 22358511 & 150 & 22358511 & 150 \\
\hline
\end{tabular}

of 12-mers due to not being able to train the 12-mer model within $11 \mathrm{~GB}$ of video RAM. The commands used have been made available on GitHub [14]. These runs were timed and resource usage was monitored during training. A general outline of the database creation process is presented in Fig. 1.

After training the neural network models, they were used to classify the previously described samples. The results were evaluated using both the necessary computing resources, which were quantified as runtime and resource usage data (memory and graphics card usage), and the classification precision, based on the 'ground truth': the theorethical composition of the reference control samples or composition identified by Kraken2 for the gut microbiome samples. We calculated the overall precision as the proportion of reads that the current tool classified the same as the 'ground truth':

$$
\text { precision }=\sum_{i=1}^{n} \min \left(r e f_{i}, t_{i}\right) \text {. }
$$


We also evaluated the overall coverage, which was achieved by each of the tools. The coverage is a relevant metric due to its ability to clearly show if a tool precision could be an artefact of it either under or over reporting when compared to other tools and was calculated as follows:

$$
\text { coverage }=\frac{\text { total reads assigned taxid }}{\text { total reads }} .
$$

Classification results (precision and coverage) were evaluated both in raw form, where the resulting taxonomic rank of the organism in the taxonomy tree was defined by the tool, and normalized in two different ways to determine the suitability of produced results for practical application: selecting the results classified at the phylum rank or at the genus rank. This was done by traversing taxonomic tree data aquired from NCBI [16].

\section{RESULTS AND DISCUSSION}

Overall training process showed high dispersion in terms of time to train/build. Kraken2 and fastDNA showed the best results (Table II). In all probability fastDNA outperformed the rest of the tools that leveraged neural networks by such a large margin because it was based on the highly efficient fastText [18]. Interestingly, there is a significant difference between GeNet and DeepMicbrobes and between MetaVW and fastDNA, even though the two pairs use similar methods when approaching the problem of learning the contents of the reference set. The disparity between MetaVW and fastDNA could be explained by the multithreaded capabilities of the tools that they are based on [12], [17]. While the disparity between GeNet and DeepMicrobes comes down to termination conditions - with DeepMicrobes terminating after 1 epoch by default [19], while the implementation of GeNet implies that it should terminate either at 400 epochs or when reaching $99.5 \%$ precision, but it did not - we stopped the training process by hand once it had reached $99.5 \%$ precision (at epoch 418) [19].

It should be noted that a signicifant portion of the training time for DeepMicrobes was spent on preprocessing the samples for further use. This along with the time that was necessary for Kraken 2 to prepare for database being built was separated out to give a better idea of the time these two tools spent on the build/train process itself (Table II).

TABLE II

TRAINING PERFORMANCE

\begin{tabular}{|l|c|c|c|}
\hline Tool & Memory usage & $\begin{array}{c}\text { Time taken } \\
\text { (including } \\
\text { preprocessing) }\end{array}$ & $\begin{array}{c}\text { Is GPU } \\
\text { accelerated }\end{array}$ \\
\hline Kraken2 & $2939 \mathrm{MB}$ & $\begin{array}{c}3 \mathrm{~m} \\
(16 \mathrm{~m})\end{array}$ & No \\
\hline MetaVW & $15.7 \mathrm{~GB}$ & $15 \mathrm{~h} 47 \mathrm{~m}$ & No \\
\hline GeNet & $2964 \mathrm{MB}$ & $10 \mathrm{~d} 23 \mathrm{~h} 50 \mathrm{~m}$ & Yes \\
\hline DeepMicrobes & $5958 \mathrm{MB}$ & $6 \mathrm{~h} 15 \mathrm{~m}$ & Yes \\
\hline fastDNA & $3205 \mathrm{MB}$ & $16 \mathrm{~h} 3 \mathrm{~m})$ & No \\
\hline
\end{tabular}

Choosing Kraken2 as the baseline when data on sample composition were not available carries a caveat: accuracies for the non-control samples are not necessarily indicative of the best performance - they are indicative of the performance most similar to Kraken2.

Some concessions had to be made when performing the classification tasks. We excluded GeNet from the classification tasks since it had no readily made classification scripts and producing such scripts was out of the scope of this article. MetaVW and fastDNA required single input files (we found functions that implied that paired end data could be fed into fastDNA, but could not find a way of accessing it without modifying the tool [20]), which meant that samples were merged using bbmerge. Additionally, DeepMicrobes could process controls as is, but required additional utility scripts to be produced to facilitate working with non-control samples due to the large amount of storage space required for the preprocessing of each sample (a 9 GB gzipped sample expanded to $127 \mathrm{~GB}$ in temporary files that resulted in a $86 \mathrm{~GB}$ tfrec file). Therefore, we batched the samples into random $10 \%$ subsamples whose results were then joined; as a result, the reported runtimes were based solely on the controls.

Just like when training the model, preprocessing samples for DeepMicrobes took a significant amount of time. It is indicated separately in the table in brackets (Table III).

TABLE III

TEST PERFORMANCE

\begin{tabular}{|l|c|c|c|}
\hline Tool & Memory usage & $\begin{array}{c}\text { Time } \\
\text { taken/sample }\end{array}$ & $\begin{array}{c}\text { Is GPU } \\
\text { accelerated }\end{array}$ \\
\hline Kraken2 & $2104 \mathrm{MB}$ & $27 \mathrm{~s}$ & No \\
\hline MetaVW & $8204 \mathrm{MB}$ & $23 \mathrm{~m}$ & No \\
\hline GeNet & - & - & - \\
\hline DeepMicrobes & $2882 \mathrm{MB}$ & $5 \mathrm{~h} \mathrm{36m}$ & Yes \\
\hline fastDNA & $3207 \mathrm{MB}$ & $24 \mathrm{~h})$ & No \\
\hline
\end{tabular}

When it comes to the amount of reads that each of the tools reported as classified, DeepMicrobes and MetaVW differentiated themselves from the rest by not reporting any results as unclassified, but always reporting some taxon ID. FastDNA fell on the other end of the spectrum classifying around $8 \%$ of the reads. We found Kraken 2 to be the only tool where the number of reads that the tool was able to classify was related to the complexity of the sample at hand: the results for control samples composed of few different species were much higher than for the diverse human samples (Table IV).

TABLE IV

Coverage

\begin{tabular}{|l|c|c|c|c|c|}
\hline Tool & $\mathrm{C} 1$ & $\mathrm{C} 2$ & $\mathrm{~S} 1$ & $\mathrm{~S} 97$ & $\mathrm{~S} 104$ \\
\hline Kraken2 & $84.94 \%$ & $82.38 \%$ & $7.95 \%$ & $6.93 \%$ & $5.24 \%$ \\
\hline MetaVW & $100 \%$ & $100 \%$ & $100 \%$ & $100 \%$ & $100 \%$ \\
\hline GeNet & - & - & - & - & - \\
\hline DeepMicrobes & $100 \%$ & $100 \%$ & $100 \%$ & $100 \%$ & $100 \%$ \\
\hline fastDNA & $8.69 \%$ & $8.60 \%$ & $8.32 \%$ & $8.43 \%$ & $8.47 \%$ \\
\hline
\end{tabular}


We found that MetaVW outperformed every other tool (even Kraken2) when examining the precision metric of the baseline results (Table V). It should be noted that these results are skewed as both Kraken2 and DeepMicrobes traverse the taxonomic tree when they are not confident enough to classify something at a certain taxonomic rank of the said tree [3], [7]. However, it is still noteworthy that MetaVW can be reasonably used to gather insight from simple samples without the need for normalization scripts.

TABLE V

PRECISION

\begin{tabular}{|l|c|c|c|c|c|}
\hline Tool & $\mathrm{C} 1$ & $\mathrm{C} 2$ & $\mathrm{~S} 1$ & $\mathrm{~S} 97$ & $\mathrm{~S} 104$ \\
\hline Kraken2 & $33.48 \%$ & $27.25 \%$ & $*$ & $*$ & $*$ \\
\hline MetaVW & $66.68 \%$ & $52.27 \%$ & $1.69 \%$ & $1.33 \%$ & $1.16 \%$ \\
\hline GeNet & - & - & - & - & - \\
\hline DeepMicrobes & $0 \%$ & $0 \%$ & $0.07 \%$ & $0.05 \%$ & $0.04 \%$ \\
\hline fastDNA & $1.01 \%$ & $1.02 \%$ & $92.13 \%$ & $92.08 \%$ & $91.86 \%$ \\
\hline
\end{tabular}

*Used as reference $(100 \%)$

When data are normalized to genus (i.e., all of the items above genus are brought down to genus rank - all others are marked as unclassified), we see some interesting patterns emerge: the reported percentages of most tools drop, with Kraken2 dropping around half of the classified reads for the non-control samples and DeepMicrobes dropping around $25 \%$ of the reads.

TABLE VI

COVERAGE GENUS

\begin{tabular}{|l|c|c|c|c|c|}
\hline Tool & $\mathrm{C} 1$ & $\mathrm{C} 2$ & $\mathrm{~S} 1$ & $\mathrm{~S} 97$ & $\mathrm{~S} 104$ \\
\hline Kraken2 & $84.21 \%$ & $81.49 \%$ & $4.02 \%$ & $3.36 \%$ & $2.54 \%$ \\
\hline MetaVW & $99.84 \%$ & $99.83 \%$ & $88.39 \%$ & $90.52 \%$ & $92.45 \%$ \\
\hline GeNet & - & - & - & - & - \\
\hline DeepMicrobes & $70.33 \%$ & $77.64 \%$ & $61.66 \%$ & $60.83 \%$ & $59.03 \%$ \\
\hline fastDNA & $8.56 \%$ & $8.65 \%$ & $8.28 \%$ & $8.39 \%$ & $8.43 \%$ \\
\hline
\end{tabular}

This increases the overall classification precision of Kraken2 up to around the performance of MetaVW with the other two tools underperforming by more than 60 percentage points when it comes to the controls. When looking at the non-control samples, interestingly, MetaVW drops below both DeepMicrobes and fasDNA in terms of precision. This can mainly be explained by the large amount of unclassified reads present in the Kraken 2 results. It is interesting to note that fastDNA performs very closely to Kraken2 when looking at the non-control samples (Table VII).

TABLE VII

PRECISION GENUS

\begin{tabular}{|l|c|c|c|c|c|}
\hline Tool & $\mathrm{C} 1$ & $\mathrm{C} 2$ & $\mathrm{~S} 1$ & $\mathrm{~S} 97$ & $\mathrm{~S} 104$ \\
\hline Kraken2 & $66.66 \%$ & $47.98 \%$ & $*$ & $*$ & $*$ \\
\hline MetaVW & $67.79 \%$ & $54.41 \%$ & $15.52 \%$ & $12.84 \%$ & $10.09 \%$ \\
\hline GeNet & - & - & - & - & - \\
\hline DeepMicrobes & $6.18 \%$ & $12.00 \%$ & $38.56 \%$ & $39.38 \%$ & $41.14 \%$ \\
\hline fastDNA & $2.38 \%$ & $2.41 \%$ & $93.03 \%$ & $93.27 \%$ & $92.68 \%$ \\
\hline
\end{tabular}

*Used as reference $(100 \%)$
Normalizing to phylum helps most of the tools retain a larger number of reads as expected since phylum is a rank that includes genus [21]. The reduction in coverage is less than one percentage point for all tools, except DeepMicrobes (Table VIII).

The pattern that the tools fall into does not differ from the results that we saw when normalizing for genus: precision improves significantly for less diverse samples and there is some improvement from baseline for other samples, although the increase is not as significant as in genus data (Table IX).

TABLE VIII

COVERAGE PHYLuM

\begin{tabular}{|l|c|c|c|c|c|}
\hline Tool & $\mathrm{C} 1$ & $\mathrm{C} 2$ & $\mathrm{~S} 1$ & $\mathrm{~S} 97$ & $\mathrm{~S} 104$ \\
\hline Kraken2 & $84.92 \%$ & $82.34 \%$ & $7.85 \%$ & $6.84 \%$ & $5.16 \%$ \\
\hline MetaVW & $100 \%$ & $100 \%$ & $100 \%$ & $100 \%$ & $100 \%$ \\
\hline GeNet & - & - & - & - & - \\
\hline DeepMicrobes & $74.23 \%$ & $80.07 \%$ & $67.81 \%$ & $67.45 \%$ & $67.96 \%$ \\
\hline fastDNA & $8.60 \%$ & $8.69 \%$ & $8.32 \%$ & $8.43 \%$ & $8.47 \%$ \\
\hline
\end{tabular}

TABLE IX

PRECISION PHYLUM

\begin{tabular}{|l|c|c|c|c|c|}
\hline Tool & $\mathrm{C} 1$ & $\mathrm{C} 2$ & $\mathrm{~S} 1$ & $\mathrm{~S} 97$ & $\mathrm{~S} 104$ \\
\hline Kraken2 & $84.91 \%$ & $72.12 \%$ & $*$ & $*$ & $*$ \\
\hline MetaVW & $92.59 \%$ & $80.20 \%$ & $7.85 \%$ & $6.84 \%$ & $5.16 \%$ \\
\hline GeNet & - & - & - & - & - \\
\hline DeepMicrobes & $36.00 \%$ & $36.00 \%$ & $33.88 \%$ & $34.22 \%$ & $33.49 \%$ \\
\hline fastDNA & $6.37 \%$ & $6.43 \%$ & $96.26 \%$ & $95.34 \%$ & $95.25 \%$ \\
\hline
\end{tabular}

*Used as reference (100\%)

The coverage of DeepMicrobes diminished significantly during normalization and this pattern was not observed in other tools. Therefore, we investigated the taxonomic division of the results for all of the tools. FastDNA provided species results, MetaVW mostly provided species results as well as sometimes responded with taxon IDs that corresponded to genotype (less than $1 \%$ of the reads). Kraken 2 traversed most of the tree with all returned taxids being valid: Kraken 2 classified around $37 \%$ of the control sample reads and around $1.4 \%$ of the non-control sample reads as species. We found that unlike Kraken2 DeepMicrobes could generate arbitrary taxon IDs that could not be found in the NCBI taxonomy data: overall DeepMicrobes returned taxon IDs that corresponded to species for around $50 \%$ of the reads and classified around $20 \%$ of the control reads, while $30 \%$ of the non-control reads had been assigned to non-existant taxon IDs [14]. The prevalence of these nonexistant taxon IDs strongly implies that the training set we used is too small for DeepMicrobes to function properly.

\section{CONCLUSION}

We found that tools leveraging deep learning still had some catching up to do when comparing their performance to Kraken2 in terms of both memory usage and runtime (with Kraken2 using less than a fifth of the memory of the most memory intensive tool and undercutting some of the tools more than 500 times when looking at runtimes at the scale that we 
tested). A notable exception when looking at the runtime was fastDNA - its performance was comparable to Kraken2. It was interesting to see that embedding based tools performed similarily to Kraken2 when evaluating their coverage and classification precision, even if this similarity in performance was limited to specific types of samples depending on the thresholding strategy that the tool employed. While the performance of DeepMicrobes did not meet the prior expectations (based on results reported in the literature) and we did not manage to fully benchmark GeNet, we still saw a lot of potential in using such tools in environments where system memory would be limited and researchers would be willing to wait for a longer amount of time to obtain the results. It is important to note that this is a statement that heavily hinges on the comparative performance of these tools when they are trained with a suitably large reference set.

When looking at the results, we can see that the most useful and operable tool is still Kraken2, but there is a disadvantage using Kraken2 with a larger database still requires a significant amount of RAM (around 200 GB [22]). This is where the deep learning based tools should readily outscale Kraken2.

We believe that a different thresholding mechanism for fastDNA could possibly make it competitive with Kraken2 even when using a small reference database such as the one that was used in this study. Exploring the behaviour of these tools with significantly larger reference databases can yield more insights into how well these tools scale with the amount of available data.

Overall, we would like to conclude that a lot of promising progress has been made when it comes to applying deep learning to bioinformatics and we are eager to see what the future will bring. We are hopeful that this paper will draw more attention to these tools and applying deep learning to bioinformatics. Additionally, we hope for someone with more complex control samples to evaluate the performance of these tools.

An interesting byproduct of performing this study is the realisation that these tools are harder to use than we had initially expected. We attempt to make replicating this study easier by outlining the setup process in the repository that contains the scripts that were used to obtain these results. This has been made publicly available on GitHub [14].

\section{ACKNOWLEDGEMENTS}

We would like to thank the Institute of Clinical and Preventitive Medicine of the University of Latvia and Latvia MGI Tech for gathering and sequencing the samples that made this article possible. The samples were sequenced within the ERDF project No. 1.1.1.1/18/A/184 “Optimisation of H.pylori Eradication Therapy for Population-Based Gastric Cancer Prevention".

\section{REFERENCES}

[1] P. Turnbaugh et al. "The Human Microbiome Project," Nature, vol. 449, pp. 804-810, 2007. https://doi.org/10.1038/nature06244

[2] E. Pasolli et al. "Extensive unexplored human microbiome diversity revealed by over 150,000 genomes from metagenomes spanning age, geography, and lifestyle," Cell, vol. 176, no. 3, pp. 649-662.e20, 2019. https://doi.org/10.1016/j.cell.2019.01.001

[3] D. E. Wood, J. Lu, \& B. Langmead, "Improved metagenomic analysis with Kraken 2," Genome Biology, vol. 20, Article no. 257, 2019. https://doi.org/10.1186/s13059-019-1891-0

[4] K. Vervier et al. "Large-scale machine learning for metagenomics sequence classification," Bioinformatics, vol. 32, no. 7, pp. 1023-1032, 2016. https://doi.org/10.1093/bioinformatics/btv683

[5] R. Menegaux and J.-P. Vert, "Continuous Embeddings of DNA Sequencing Reads and Application to Metagenomics. J. Comput. Biol., vol. 26, no. 6, pp. 509-518, June 2019. https://doi.org/10.1089/cmb.2018.0174

[6] M. Rojas-Carulla et al. "GeNet: Deep Representations for Metagenomics," bioRXiv, preprint. Available: https://doi.org/10.1101/537795

[7] Q. Liang et al. "DeepMicrobes: taxonomic classification for metagenomics with deep learning," NAR Genomics \& Bioinformatics, vol. 2, no. 1, 2020. https://doi.org/10.1093/nargab/lqaa009

[8] S. Mangul et al. "Systematic benchmarking of omics computational tools," Nat. Commun., vol. 10, Art. no. 1393, 2019. https://doi.org/10.1038/s41467-019-09406-4

[9] P. P. Gardner et al. "A meta-analysis of bioinformatics software benchmarksreveals reveals that publication-bias unduly influences software accuracy," bioRxiv, preprint. Available: https://doi.org/10.1101/092205

[10] MGI DNBSEQ-T7 [Online]. Available:

https://en.mgitech.cn/products/instruments_info/5/[Accessed September 2020]

[11] Zymo control sample documentation [Online]. Available: https://files.zymoresearch.com/protocols/_d6300_zymobiomics_microbi al_community_standard.pdf [Accessed Aügust 2020]

[12] Vowpal Wabbit documentation [Online]. Available: https:/github.com/VowpalWabbit/vowpal_wabbit/wiki [Accessed September 2020]

[13] MetaVW data store [Online]. Available: http://cbio.mines-paristech.fr/largescalemetagenomics/large-scalemetagenomics-1.0.tar.gz [Accessed August 2020]

[14] GitHub repository [Online]. Available: https://github.com/lucren/itms bio ml 2020 [Accessed September 2020]

[15] R. Maier, R. Zimmer, \& R. Küffner, "A Turing test for artificial expression data," Bioinformatics, vol. 29, no. 10, pp. 2603-2609, 2013. https://doi.org/10.1093/bioinformatics/btt438

[16] NCBI taxonomic data [Online]. Available: ftp://ftp.ncbi.nih.gov/pub/taxonomy/taxdump.tar.gz [Accessed July 2020]

[17] A. Joulin, E. Grave, P. Bojanowski and T. Mikolov, "Bag of tricks for efficient text classification," arXiv preprint 1607.01759, 2016

[18] DeepMicrobes documentation [Online]. Available: https://github.com/MicrobeLab/DeepMicrobes/blob/master/document/tra in.md [Accessed August 2020]

[19] GeNet implementation [Online]. Available: https://github.com/lucren/GeNet/blob/master/code/genet train.py [Accessed September 2020]

[20] fastDna paired-end functionality [Online]. Available: https://github.com/rmenegaux/fastDNA/blob/b4aa88cf95e50e38d35e996 b1a0b4a8b626f2fee/src/model.cc [Accessed August 2020]

[21] IAPT taxonomic nomenclature [Online]. Available: https://www.iapttaxon.org/nomen/main.php?page=art3[Accessed September 2020]

[22] Kraken2 manual [Online]. Available: https://github.com/DerrickWood/kraken2/wiki/Manual [Accessed September 2020] 
Kristaps Bebris currently holds a Master degree with specialisation in bioinformatics from Uppsala University, from which he graduated in 2017. $\mathrm{He}$ is currently a Research Assistant at the University of Latvia, Data Scientist at Sannsyn Latvia and a PhD student at Riga Technical University. His main interests are natural language processing, bioinformatics and genetic algorithms.

E-mail: kbebris@edu.lu.lv

Inese Polaka received her Doctoral degree (Dr. sc. ing.) in information technology from Riga Technical University in 2014. She has since worked at Riga Technical University and holds the position of Assistant Professor at the moment. Her fields of interest are machine learning, data mining, especially in medical applications, evolutionary algorithms, transparent and interpretable supervised and unsupervised learning methods, as well as bioinformatics (main focus on prokaryotic metataxonomics and metagenomics).

E-mail: inese.polaka@rtu.lv

ORCID iD: https://orcid.org/0000-0002-9892-7765 